Meta

Journal des traducteurs

Translators' Journal

\title{
Le cas du traducteur fonctionnaire
}

\section{Denys Goulet}

Volume 11, numéro 4, décembre 1966

La formation du traducteur

URI : https://id.erudit.org/iderudit/002956ar

DOI : https://doi.org/10.7202/002956ar

Aller au sommaire du numéro

Éditeur(s)

Les Presses de l'Université de Montréal

ISSN

0026-0452 (imprimé)

1492-1421 (numérique)

Découvrir la revue

Citer cet article

Goulet, D. (1966). Le cas du traducteur fonctionnaire. Meta, 11(4), 127-138.

https://doi.org/10.7202/002956ar d'utilisation que vous pouvez consulter en ligne.

https://apropos.erudit.org/fr/usagers/politique-dutilisation/ 


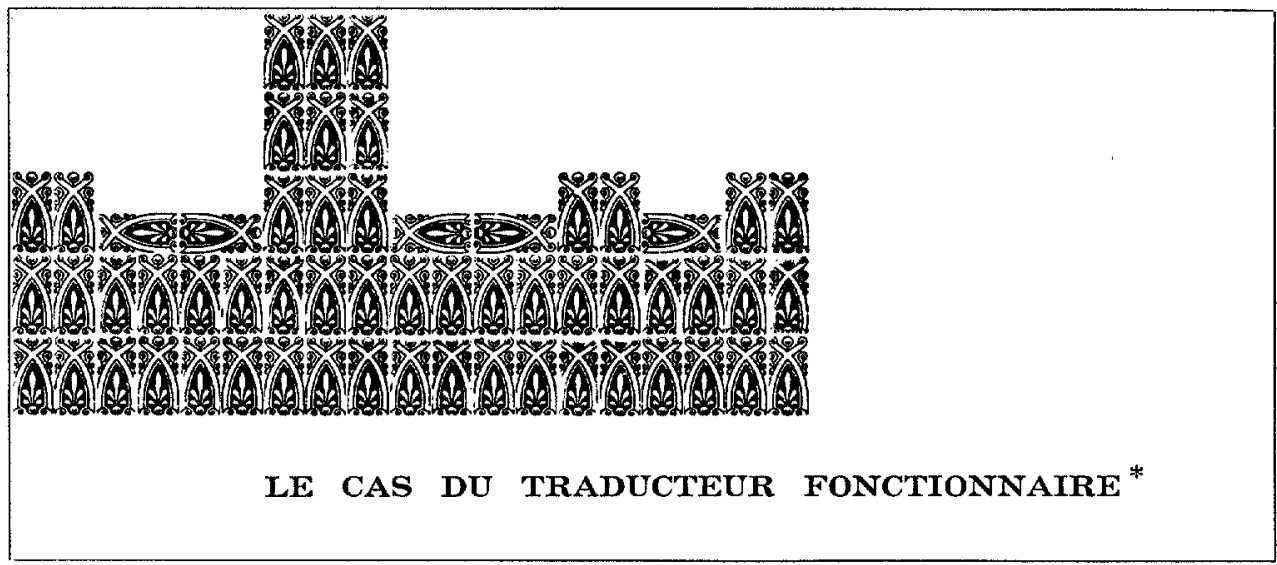

DePuIs une trentaine d'années que je travaille à Ottawa, je constate que la plupart des fonctionnaires qui y exercent ou y ont exercé le métier de traducteur sont entrés dans la carrière fortuitement. Fortuitement en ce sens qu'aucun d'entre eux, que je sache, ne s'était systématiquement préparé, dès ses primes années, dès l'école ou le collège, à devenir traducteur, comme maints jeunes gens, touchant au terme de leur scolarité, décident un jour, par vocation ou par tempérament, de se faire agronome, architecte, chimiste, comptable, dentiste, homme de loi, ingénieur, instituteur, prêtre ou médecin.

Les traducteurs fonctionnaires sont le plus souvent des gens qui, ayant manifesté au collège certaines dispositions pour la version ou le thème, et ayant habituellement recueilli de bonnes notes dans leurs exercices d'anglais, ont de la sorte acquis des notions fondamentales du métier (vocabulaire, grammaire, stylistique) et, partant, se sont un beau jour avisés de se présenter à un concours comportant l'épreuve écrite d'admission à un poste de traducteur de textes officiels. Beaucoup d'entre eux, anciens «forts en version » ou «premiers en narration », s'étaient d'aborrd orientés vers un emploi qui leur offrait quelque occasion de tirer parti de leur aptitude, innée ou acquise, à s'exprimer oralement ou par écrit cum laude, magnia cum laude, ou summa cum laude. En effet, il y eut une époque où, dans l'administration, à Ottawa, la plupart des traducteurs étaient d'anciens journalistes, instituteurs ou séminaristes ${ }^{1}$ recrutés à peu près exclusivement, et pour cause, dans la province de Québec. Or, du fait qu'ils avaient «manié la plume » pour le compte de quelque journal à grand ou à faible tirage, ou enseigné la rédaction au niveau scolaire ou collégial, ces gens possédaient au départ un bagage plus ou moins appréciable de procédés expressifs leur permettant de traduire en français, sans difficultés insurmontables, tout ce que pouvaient contenir les textes établis en anglais administratif ou technique (correspondance, formules, rapports, monographies), ou en anglais universel et à tous les niveaux (débats des Communes et du Sénat, ou procès-verbaux des délibérations de comités de la Chambre). Aucun d'eux, à ma connaissance, n'avait suivi un «cours » de traduction pour la manifeste raison que les maisons d'enseignement ne dispensaient pas de tels cours.

* Partout dans le présent article, les italiques sont introduites par l'auteur.

1. Certains avaient exercé quelque autre profession: agronome, chimiste, comptable, homme de loi, libraire, médecin, physicien, publicitaire, etc. 
La formation s'opérait donc à pied d'œuvre, par tâtonnements et suivant le procédé classique de la revision, où le débutant remet son travail à un traducteur expérimenté qui s'applique à en supprimer tout d'abord les contresens (péchés mortels du traducteur), les solécismes, les fautes contre l'usage et, le cas échéant, les fautes d'orthographe. La méthode empirique, quoi.

Durant les années dont je parle, personne encore n'avait entrepris de rédiger ce qui aurait pu être considéré comme un «manuel» ou «traité » de traduction. Certes il existait déjà quelques ouvrages didactiques, tels les Traquenards de la version anglaise, de Veslot et Banchet (Hachette, 1922) et son pendant, les Épines du thème anglais, de Veslot (Hachette, 1892), mais ces ouvrages s'adressaient « aux seuls élèves d'anglais des lycées et collèges ... et aux aspirants au baccalauréat ». Or peu de traducteurs possédaient ces ouvrages et beaucoup, assurément, n'en soupçonnaient même pas l'existence.

Le traducteur fonctionnaire se débrouillait donc au moyen de son acquis personnel, diversement opulent, et utilisait pour son entendement de la langue de départ, sa «langue seconde », un savoir que, en général, il avait puisé, jeune encore, dans les 300 pages du Nouveau cours de langue anglaise selon la méthode d'Ollendorff (Montréal, Beauchemin), ouvrage approuvé par le Conseil de I'Instruction publique de la province de Québec le 12 mai 1909 et «Enregistré conformément à l'acte du Parlement du Canada, en l'année mil huit cent soixantehuit, par M. Ant. Nantel, Ptre., au bureau du ministre de l'Agriculture ». C'était un manuel «partout répandu ... [et] qui a fait ses preuves dans le passé », disait dans sa préface Monsieur Olivier Maureault, P.s.s., recteur de l'Université de Montréal. Ce cher Nouveau cours, je n'ai pas manqué de m'y tremper moi aussi car, « partout répandu », Ollendorff était prescrit dans les classes de propédeutique au Collège de Saint-Boniface, vers 1914. Encore dans l'édition revisée en 1935 par quatre professeurs canadiens (dont trois anglophones) on trouve des exemples aussi plausibles que ceux-ci:

Avez-vous l'aiguille et le fil du tailleur? J'ai son aiguille mais je n'ai pas son fil.

Avez-vous le chapeau de l'aveugle?

Où allâtes-vous hier au soir?

Je m'habille: I dress myself.

The English have large business.

Qu'elle se hâte de s'habiller: Let her make haste to dress herself.

Aviez-vous dîné quand je suis entré ?: Had you dined when I came in?

Est-ce à moi que vous parlez ?: Is it to me (that) you speak?

On discerne dans ces tournures une imparfaite réussite de l'《équivalence figée $\gg^{2}$ et on les juge moins idiomatiques que celles qui figurent dans des manuels scolaires plus récents; mais il reste que, dans l'ensemble, le vénérable manuel d'Ollendorff a été un instrument d'initiation très utile, car il a enseigné à maintes générations de Canadiens français à conjuguer des verbes anglais aux formes affirmative, négative, interrogative, et à insérer convenablement, suivant les canons de l'usage, les pronoms sujets ou compléments. Or, comme l'affirmait, avec raison croyonsnous, le professeur W. Conacher, de Toronto, en 1920: «A working knowledge of the verb is by far the most important consideration in starting to speak a

2. J.-P. Vinay et J. Darbelnet, Stylistique comparée du français et de l'anglais, nouv. éd., Montréal, Beauchemin, 1960 , p. 240. 
foreign language. Mistakes in the gender of nouns or adjectives do not cause the deadlock that the inability to «predicate » a thing does.»C'est ce qui parait expliquer pourquoi tant de compatriotes anglophones qui s'inscrivent adultes à des cours de français abandonnent désespérément la partie lorsqu'il s'agit pour eux. non plus de retenir des noms d'objets ( la plume de ma tante; le perroquet du jardinier; la montre du maître de musique ») mais de conjuguer à tous les modes et à tous les temps - y compris l'imparfait du subjonctif - les verbes pronominaux et irréguliers du succulent et nerveux parler de France. Que nombre d'entre eux tout de même y parviennent, c'est admirable et digne de tous les encouragements; mais s'ils réussissent, n'est-ce pas surtout parce qu'ils possèdent ce que Stephen Leacock appellera « une certaine aptitude », un certain talent ?

Et voici qu'entrent en scène les mots fatidiques: culture et talent. Certes les meilleurs traducteurs fonctionnaires que $\mathrm{j}$ 'ai connus au cours des trente dernières années étaient des hommes ou des femmes qui, d'entrée de jeu, justifiaient d'une culture générale parfois très étendue, voire impressionnante ${ }^{3}$. C'étaient des gens d'une vive curiosité intellectuelle, lisant avidement de tout, et qui s'étaient formés eux-mêmes ${ }^{4}$ par un recours instinctif à tout ce que la page imprimée pouvait offrir de complément aux seuls dictionnaires, ces béquilles ${ }^{5}$.

Notons aussi que, au cours des années 1920 à 1940, la plupart des traducteurs de l'État possédaient, pour l'avoir accumulé à l'école et au collège, ou sous l'effet de lectures personnelles, un commun bagage de modèles et de souvenirs «que chaque Français porte dans sa giberne, avec le bâton de maréchal ». Chacun retenait des mots, des phrases, des vers, des tournures qui se retrouvent dans les livres de lecture, grammaires et manuels d'exercices de quiconque a fréquenté les écoles communales de France ou, ai-je lieu de croire, les écoles et collèges du Québec. Le palmarès de ces souvenirs, ancrés dans le subconscient et, partant, à tout jamais normatifs sous le rapport du goût et de la forme, a été magnifiquement présenté par Gaston Bonheur dans Qui a cassé le vase de Soissons? (Laffont, 1963). Je ne saurais indiquer avec assurance quelles ont été les lectures françaises formatrices qui ont été en quelque sorte imposées à tous les écoliers de la province de Québec depuis le début du siècle, étant donné que, pour ma part, c'est dans une autre province que $\mathrm{j}$ 'ai appris à parler et à lire le français; $j$ 'ai cependant constaté qu'à Ottawa mes collègues originaires du Québec s'étaient abreuvés, dans leur jeunesse, aux mêmes sources littéraires que les Français d'outremer.

Pour la même raison, je ne sais dans quels manuels mes collègues qui ont été instruits dans le Québec ont étudié la grammaire française. Au Manitoba, on a utilisé, suivant les époques et les institutions, divers manuels: Pierre Larousse,

3. Il se trouvait parmi eux des écrivains, des poètes, des sociologues, des universitaires.

4. « J'ai bien l'impression que presque tous les traducteurs canadiens en exercice se sont formés eux-mêmes, tout en apprenant leur métier. C'est d'ailleurs tout à leur honneur d'avoir, en général, si bien réussi. Mais faut-it qu'il en soit toujours ainsi? (J.-P. Vinay, Journal des Traducteurs, vol. $8, \mathrm{n}^{\circ} 4,1963$, p. 113.)

5. "Mal utilisé, le dictionnaire peut être L'ENNEMI du traducteur. Le mot juste ne se découvre qu'en cernant le sens exact du mot à traduire, en effectuant des allers et retours du dictionnaire de thème au dictionnaire de version, ou mieux, en utilisant un dictionnaire tout anglais. Ne vouloir se servir que d'une partie du dictionnaire, c'est vouloir marcher sur une jambe." (L. Guierre, Le Mot juste, Paris, Vuibert, 1959, p. 7.) "... le Dictionnaire, véritable armoire aux poisons, mortelle à qui ne sait pas lire". (Georges Lafour. cade, Textes de traduction, Paris, Didier, 1963, p. 6.) 
Claude Augé, E. Ragon, Larive et Fleury, Noël et Chapsal, Brachet et Dussouchet, ainsi que des manuels édités au Canada: Frères des Écoles chrétiennes, Frères du Sacré-Cour, E. Robert, c.s.v., etc. Cependant, la grammaire que j'ai le mieux connue et appréciée, et que j'ai encore aujourd'hui par-devers moi, c'est celle qu'un distingué journaliste et polémiste montréalais avait un jour qualifiée de «pieuse » grammaire (Cours supérieur et Exercices), œuvre de l'abbé A. Aubert, professeur au Séminaire de Québec, «ouvrage approuvé par le Comité catholique de l'Instruction publique, le 25 septembre 1912 » (Québec, L'Action sociale Ltée, 1917). Le préfacier, M. Adjutor Rivard, précise qu'il s'agit ici de l'« œuvre d'un grammairien et non d'un grammatiste ${ }^{6} \%$. Ternement parée de bougran cendre bleue, mais superbement imprimée et indexée - et, par cela même, commode à consulter - cette grammaire de 350 pages, je l'ai trouvée bien pratique. Durant près de cinquante ans, elle m'a été la clé de tous problèmes courants d'ordre grammatical. Sans doute l'estimerait-on aujourd'hui fort dépassée - elle présente encore quatre conjugaisons au lieu des trois «groupes» de verbes - mais, ne serait-ce que pour la liste qu'elle donne (en 26 pages) de «locutions vicieuses» à corriger et particulièrement courantes au Canada, j'y vois encore un guide sûr pour le Canadien soucieux d'une honnête correction dans ses écrits.

Bien entendu, par souci professionnel, un traducteur tiendra aujourd'hui à se munir d'un ouvrage plus représentatif de l'« usage contemporain » et c'est ainsi que, pour ma part, je me suis procuré ultérieurement diverses autres grammaires, par exemple celles d'Abel Hermant (1932) ${ }^{7}$; Michaut et Schricke (1934); Larousse du XXe siècle (1936); Maurice Rat (1946); Albert Dauzat (1947); Galichet (1950) (1964) (1965); Maurice Grevisse ( $5^{\circ}$ éd., 1953) « à mi-chemin entre les traditionalistes têtus et les novateurs dangereux $»$, tribunal de dernière instance dans les causes délicates et bilieusement contestées; et puis, récemment, la Grammaire Larousse du français contemporain (1964) où les quatre auteurs ont « cherché à concilier l'abondance des informations, l'emprunt aux méthodes les plus récentes, avec le souci de l'organisation et de la plus grande clarté ». Elle règle en 27 mots la question de l'emploi de l'imparfait du subjonctif et elle a 19 pages d'index, sur deux colonnes par page. Il n'y est plus question d'« analyse logique » mais de «la phrase simple » et «la phrase complexe». Quoiqu'elle donne encore d'《assez nombreuses références au français classique », elle cite copieusement les plus notables tisserands de la prose française contemporaine, de si loin que ceux-ci puissent s'éloigner de la prose flaubertienne ou anatole-française, réputée passable.

Si tant est qu'il soit permis de prétendre que le manuel scolaire - en particulier la grammaire — joue un rôle clé dans l'acquis linguistique d'un traducteur, fonctionnaire ou autre ( Dis-moi qui tu as hanté...»), il me semble que ce pourrait être non seulement intéressant mais utile de savoir dans quels manuels les traducteurs ont étudié la grammaire française. Il y a, à ce propos, de fort

6. Mot admis par l'Académie en 1798 (Hatzfeld-Darmesteter). Ne figure pas au Petit Larousse 1966, mais y était encore en 1952.

7. Grammaire dite "de l'Académie ", qui n'a probablement jamais servi à personne, pour les multiples motifs que Ferdinand Brunot a subséquemment fait valoir dans ses (260) Observations sur la Grammaire de l'Académie, Paris, Droz, 1932. 
pertinentes remarques aux alinéas 616 et 617 du tome II-B du rapport Parent. Un recensement en ce sens permettrait peut-être de dégager une conclusion éclairée quant aux manuels qu'il conviendrait de prescrire désormais aux aspirants traducteurs en tant qu'éléments indispensables d'une solide formation. N'y aurait-il pas moyen ainsi de dresser une liste d'ouvrages jugés utiles ou essentiels à cette fin précise? C'est une tâche que mènerait sûrement à bien une commission ad hoc, ou un organisme tel que la STIC ou l'ATIO, dont l'action puisse librement s'exercer au-delà de toute frontière. J'y entrevois, pour ma part, un facteur de progrès. En effet, je crois pouvoir affirmer que les éléments de force du traducteur dit «chevronné », d'élite si l'on veut, ce ne sont pas la grammaire et la stylistique qu'il a scolairement étudiées il y a cinq, dix et surtout vingt ans, mais celles qu'il a réapprises au cours de son travail actuel.

Disons, au surplus, que rien ne sert d'avoir des brassées de manuels si on ne les ouvre pas souvent, même quotidiennement, ne serait-ce que pour tonifier son savoir ou pour cultiver l'infiniment précieux penchant que mon ami et collègue aujourd'hui retraité, Louis-Philippe Gagnon, appelait, avec mélancolique compassion, « le doute salutaire ». Un peu d'humilité, quoi, devant les multiples registres, la plantureuse diversité du langage français ${ }^{8}$.

Autour des années trente, le traducteur fonctionnaire, à Ottawa, se tirait d'affaire avec les dictionnaires bilingues d'alors, type Cassell, ou Spiers et Surenne (plus développé mais qui datait déjà) ou avec 1'estimable Elwall ${ }^{9}$, mais surtout, surtout à l'aide des Clifton-Grimaux: anglais-français 1351 pages, français-anglais 1237 pages, édités à Paris chez Garnier, le premier en 1914, le second en 1923. L'un et l'autre de ceux-ci avaient d'ailleurs été « entièrement refondus et considérablement augmentés » par $\mathrm{J}$. McLaughlin, officier de 1'Instruction publique, etc. Le Clifton-Grimaux-McLaughlin se disait, avec raison d'ailleurs, "plus complet » que tout autre dictionnaire du temps, et comprenait « un nombre considérable de termes spéciaux relatifs aux arts et aux sciences, au commerce et à l'industrie, qu'on chercherait vainement dans les ouvrages antérieurs ... $\gg$. C'est ainsi que le Clifton-Grimaux-McLaughlin a été le dépanneur classique du traducteur de l'État jusqu'à ce que parût, en 1934, l'imposant Mansion (communément dénommé Harrap, ou plus souvent «Arrapsse ») français-anglais, suivi en 1939 de son congénère best-seller anglais-français, qui eurent tôt fait de supplanter le Clifton.

En 1934 également, Hachette avait édité un remarquable nouveau dictionnaire manuel anglais-français ${ }^{a}$ que venait d'établir Charles Petit, professeur agrégé

8. «La langue française est réputée pour sa clarté. Entendons-nous bien: la langue française comporie en soi tout ce qu'il faut aux esprits clairs pour s'exprimer clairement. Elle comporte aussi tout ce qu'il faut pour embarrasser et perdre les esprits confus. Elle est pleine de ressources et d'embûches. Elle est pareille à ces chevaux de race qui, montés par un bon cavalier, font des prodiges, et qui trébuchent à chaque pas entre les mains d'un incapable. 》 (Georges Duhamel, Discours aux nuages, p. 32.)

9. Qui cite en page de titre cette réflexion qu'il attribue à Andrieux mais qui est de Samuel Johnson (1755): « Tous les autres auteurs peuvent aspirer à la louange; les lexicographes ne peuvent aspirer qu'à échapper aux reproches. \Il m'a toujours semblé que la même réflexion s'applique aux traducteurs.

9a. « Conçu à la fois pour servir d'instrument de travail aux élèves des lycécs et collèges et aux étudiants des Facultés qui se consacrent à l'étude de la langue et de la littérature anglaise, et pour répondre aux besoins du grand public ... [Ses définitions ou traductions sont] animées et précisées par d'abondants exemples qui éclairent les différents sens des mots avec toutes leurs nuances et donnent leur vraie valeur aux idiotismes et aux locutions courantes... * 
d'anglais au Lycée Buffon, en s'inspirant de certaines caractéristiques du prestigieux Concise Oxford des frères Fowler, ces géants. M. Petit étant décédé en 1940, la contrepartie anglaise-française de son excellent dictionnaire ne parut qu'en 1946, achevée par un sien collègue, le professeur W. Savage.

C'est aussi en 1934 que parut le Concise Oxford French-English Dictionary, cuvre d'Abel et Marguerite Chevalley, universitaires parisiens. Inspiré lui aussi de la méthode fowlérienne, ce modeste ${ }^{10}$ et méticuleux ouvrage renferme, dans son Introduction, un exposé à la fois lumineux et précis des principes régissant la bonne lexicographie bilingue mais aussi la traduction. $\grave{A}$ mon avis, la lecture attentive, et non accélérée, de ces dix pages où se trouve la quintessence de la matière, devrait être obligatoire pour tout traducteur, aspirant ou praticien.

Toutefois, en raison de leur nomenclature plus restreinte, ni le Charles Petit (50 000 mots) ni le Chevalley (40000 mots) ne pouvaient prétendre, en tant qu'outils du traducteur professionnel, à déloger le Mansion que venait de lancer la maison Harrap, de Londres.

Accessoirement, les traducteurs de l'État disposaient d'un certain nombre de dictionnaires techniques spécialisés (Barrère, Betesta, Duden, Kettridge, Malgorn, Pagé, Plattard, Plumon, Schlomann, Sell, Tolhausen, Travers, Vivant, Wilcox, etc.). Mais, même avant la seconde Grande Guerre, les dictionnaires techniques, généraux ou spécialisés, ne pouvaient longtemps rester à jour. On ne pouvait compter y trouver les centaines de termes nouveaux qui entraient dans l'usage en raison des progrès de la technique dans de nombreux domaines, v.g.:

l’aviation (pulso-, strato-, et turbo-réacteurs, moteurs-fusées, autoguidage, téléguidage);

la physique et la chimie (missiles, fusées-engins, roquettes, projectiles autocommandés ou téléguidés, photocopieuses, laser, maser; rayonne, nylon, dacron, orlon, térylène, détergents ou détersifs, enduits acryliques, butadiène, polymères, isorel, masonite, matières plastiques et produits cellulosiques, D.D.T., L.S.D., antibiotiques, pénicilline, sulfamides);

l'électronique (radar, sonar, téléviseurs, magnétophones, transistors, servomécanismes, automation);

la cinématographie et la photographie (cinérama, vistavision, procédé polaroïd, flashes, objectifs zoom - à focale variable, fluoruration des lentilles);

l'astronautique et le vocabulaire engendré par la mise sur orbite de tant de satellites artificiels depuis le lancement de Spoutnik I à l'automne de 1957; l'informatique, le langage électronique; et le resté, et le reste...

À ce propos, il importe de se rendre compte qu'à partir des années quarante, et singulièrement depuis le 16 juillet 1945 (l'expérience d'Alamogordo), deux événements se sont produits qui sont assez littéralement formidables pour constituer des tournants dans l'histoire de l'humanité: la mise au point de la bombe atomique et la généralisation des ordinateurs électroniques. Il n'y a plus moyen de douter que les ordinateurs de demain vont révolutionner les communications, le commerce, la science et puis aussi l'éducation, de mille façons encore à peine entrevues.

Une des conséquences immédiates, c'est que la prolifération des termes techniques est devenue telle que les éditeurs ont peine à pourvoir à la criante demande de nouveaux dictionnaires, lexiques et glossaires spéciaux, bilingues ou multilingues.

10. "Its main and special purpose, comparatively unambitious, is to prove more incegrally helpful to students and translators than some existing works of the same class. " (Préface du Concise Oxford French-English Dictionary, p. Ir.) 
Des compilateurs canadiens se sont mis de la partie et déjà, de ce côté-là, nous disposons de divers recueils d'expressions techniques, par exemple ceux de MM. René Dupuis, Lucien Hudon, Marcel Lefebvre, Lucien Normandeau et Gérard Proulx. Du reste, les ouvrages de cette nature se multiplient et les listes bibliographiques en signalent presque mensuellement de nouveaux. Sans parler de ceux qui émanent de France (éditions Dunod, Eyrolles, etc.) ou d'Angleterre (collection Pergamon), signalons que, depuis moins de dix ans, la maison Elsevier (Amsterdam) en a, à elle seule, édité près de quatre-vingts.

Cependant, si l'accès à la terminologie technique la plus récente ne peut que faciliter la tâche du traducteur qui soit souvent appelé à s'en servir, la qualité de sa traduction dépend en premier lieu de tout autre chose: l'étendue de sa connaissance des deux langues de travail, et la capacité de s'exprimer correctement. Sur le plan de la correction du langage et du bon usage, le traducteur fonctionnaire avait pu recourir à divers ouvrages canadiens ou français, parfois fort développés, parfois consistant en de simples plaquettes:

1880-Manuel des expressions vicieuses les plus fréquentes, par F. Gingras, traducteur aux Communes.

1880 - Glossaire franco-canadien et vocabulaire de locutions vicieuses usitées au Canada, par Oscar Dunn.

1886 - Petit dictionnaire raisonné des difficultés et exceptions de la langue française, par Soulice et Sardou.

1888 - Anglicismes et canadianismes, par Arthur Buies.

1896 - Dictionnaire de nos fautes contre la langue française, par Raoul Rinfret.

1914 - Glossaire de la Société du Parler français au Canada.

1920 - Dictionnaire des anglicismes, par Edouard Bonnafé.

1924 - Zigzags autour de nos parlers, $1^{\text {re }}$ série, par Louis-Philippe Geoffrion; $2^{\text {e }}$ série en $1925 ; 3^{\mathrm{e}}$ série en 1927.

1927 - Dictionnaire du bon langage, de l'abbé É̂ienne Blanchard, qui avait aussi publié En garde!, En français, Le Bon Français en affaires, Mille mots illustrés, etc.

1928 - Les Faux Amis, ou Les pièges du vocabulaire anglais, de Koessler et Derocquigny.

1930 - Le Vrai Ami du traducteur anglais-français, de Félix (de Grand Combe) Boillot.

1930 - Guide du Traducteur des Débats, de Wilfrid Gascon.

1931 - L'Expression juste en traduction ( $1^{\text {re }}$ série); Questions de langage ( $2^{\mathrm{e}}$ série); Traduction (1941); Langage et Traduction (1961), par Pierre Daviault.

1932 - Autour de notre français, de L.-P. Geoffrion.

1936 - Les Etrangers dans la cité, de Léon Lorrain.

1936 - Expressions justes en affaires, d'Adjutor Fradet.

1937 - Duden français et Duden anglais.

1937 - Vocabulaire pratique de l'anglais au français, de Léon Gérin.

1938 - Fragments lexicographiques, de René de la Durantaye.

1939 - Le Ramage de mon pays, par Victor Barbeau.

1945 - Dictionnaire militaire anglais-français, français-anglais, "publié sous la direction du chef d'état-major général, Canada ".

1948 - Ecrasons le perroquet, par Louvigny de Montigny.

1951 - Dictionnaire des gallicismes, d'Elisabeth Pradez.

1956 - Le Second Vrai Ami du traducteur, par Félix Boillot.

(Cette liste n'étant pas exhaustive, je saurais gré à quiconque me signalerait toute lacune notoire.)

En 1951, l'Association technologique de langue française d'Ottawa, fondée en 1920, lançait un Bulletin trimestriel ${ }^{11}$ dont, jusqu'à décembre 1957, elle allait publier 26 numéros. On y trouvait une abondance de notes et observations, et de

11. Dans sa lettre du 26 février 1951 à M. Augustin Poivin, directeur du Bulletin, M. Markland Smith, président de l'Association, disait: "L'Association technologique a depuis trente ans, même au dire des étrangers, fait un extraordinaire travail d'épuration et d'uniformisation des termes officiels du gouvernement. Voici qu'un bulletin, no:re bulletin. nous met en relations plus étroites les uns avec les autres pour nous permettre de mieux collaborer et de nous éclairer mutuellement. Soyons-en reconnaissants et profitons le plus possible de ce moyen d'action. " 
très utiles chroniques et glossaires, fruits de la collaboration bénévole de traducteurs épris de recherches et de perfectionnement. Conscient de la valeur pratique de pareille formule, le Bureau des traductions institua en 1954 un Service de terminologie qui, jusqu'à ce jour, a publié 123 Bulletins et 37 Instructions terminologiques.

Évidemment, glossaires et lexiques peuvent fournir la matière première: les termes; mais dans toute traduction il y a la matière et il y a la forme. Sous le rapport du style ${ }^{12}$, un traducteur travaille selon les normes qu'il a épousées par suite de sa formation première, de son goût, de ses lectures. Or, suivant son tempérament, il peut voir dans chaque nouvelle tâche qui lui est assignée, ou bien une corvée dont il ne songera qu'à se débarrasser au plus tôt et avec un minimum d'effort, ou bien une nouvelle occasion de s'exercer à rédiger le plus correctement et le plus simplement possible ${ }^{13}$. Surtout à ses débuts dans le métier, un grand nombre des retouches ou « revisions » apportées à ses textes lui paraissent immotivées, arbitraires ou franchement insensées (euphémisme), mais, chose étrange, avec le temps elles lui semblent «parfois» (tout le monde peut se tromper, que diable !) ou même « assez souvent » admissibles, judicieuses, voire indispensables. À la fin, il ne lui arrive plus que de commettre des «fautes d'inadvertance $\gg$ ou des lapsus calami, ces dictames de l'amour-propre contusionné ou simplement éraflé. Et pourtant, se dit-on quand on y pense, il y a les manuels: ouvrages normatifs, bourrés de conseils, qui se vendent dans les librairies et dont chacun a été composé, en général, par quelqu'un qui s'y connaît à l'intention... des autres.

Bien sûr, il existe depuis longtemps des manuels de style, mais alors que naguère on ne trouvait sur place que les ouvrages d'Antoine Albalat et d'Eugène Legrand, depuis quelques années les traités de stylistique et guides du bon usage foisonnent. C'est tout un pactole aujourd'hui pour qui aspire à la correction, voire à l'élégance de la forme. Que de richesses à la portée de l'artisan résolu de lire, relire, noter, étudier, retenir et utiliser !

Parmi les plus prestigieux manuels français se rangent ceux de Charles Bally, Ch. Bruneau, F. Brunot, Marcel Cohen, Marcel Cressot, Albert Dauzat, René Georgin, Henri Godin, Georges Gougenheim, Remy de Gourmont, Maurice Grevisse, Abel Hermant, Jean Humbert, R. Le Bidois, J. Marouzeau, Antoine Meillet, Maurice Rat, Ferdinand de Saussure, Aurélien Sauvageot, André Thérive et $\mathbf{J}$. Vendryès.

Et il y en a bien d'autres qui, vraiment, mériteraient d'être mieux connus; ceux, par exemple, de H. Brémond, Robert Catherine, Pierre Clarac, Paul Comiant, Gilbert Corot, M. Courault, Fernand Desonay, Camille Dudan, Gommaire Dyk-

12. "... Cette libération de la pensée et cette expansion de la personne au moyen de l'expression verbale atteignent parfois, par leur contenu et par la forme même conf́érée à la langue - qui devient alors style - le niveau d'une véritable création, susceptible de rejoindre et de modifier l'âme et l'intelligence des autres hommes. A cause de cette triple fonction: représentation, expression, création, le langage constitue l'un des éléments les plus significatifs de la personnalité. » (Rapport [Parent] de la Commission royale d'enquête sur l'enseignement dans la province de Québec, Deuxième partie, t. II [suite], 1964, p. 24, art. 572.) * La langue est gouvernée par des valeurs psychologiques qui créent et recréent sans cesse la valeur fiduciaire des mots. " (Georges Galichet, Essai de grammaire psychologique, Paris, P.U.F., 1950, p. Xv.)

13. Cf. René Georgin, L'Inflation du style, Paris, Les Editions sociales françaises, 1963. 
mans, René Étiemble, Fernand Feugère, Henri Fontenay, Frey et Guenot, Marcel Galliot, Lionel Guierre, M. Guiraud, J. Hanse, G. Lanson, Étienne LeGal, André Martinet, Arthur Masson, Henri Mitterand, André Moufflet, Georges Mounin, Louis Piéchaud, Ernest Rogivue, Sapir, Maurice Schöne et Arsène Soreil. À quoi il convient, certes, d'ajouter les réalisations de censeurs et stylisticiens canadiens: Victor Barbeau, René de Chantal, Jacques Clément, Gérard Dagenais, Jean Darbelnet, Pierre Daviault, Léon Gérin, Jean-Marie Laurence, Paul Morin, Jean-Paul Vinay. (Pour les titres de la plupart de ces ouvrages, dont le Centre de terminologie va bientôt publier une liste, voir la section bibliographique de Grevisse, ou les rayons de quelque bonne bibliothèque municipale ou universitaire.)

Chose certaine, tout traducteur, débutant ou vétéran, trouvera dans de tels manuels (ou dans la seule série des ouvrages de René Georgin) les éléments d'une formation personnelle de plus en plus poussée, des guides qui le conduiront très loin dans les voies montantes, sablonneuses, du bien-dire et du mieuxdire.

Voilà pour ces assises de la formation du traducteur: la grammaire, la stylistique, le bon usage. Cependant, comme le fait observer Georges Mounin: «J.-P. Vinay et J. Darbelnet ont été les premiers à s'être proposé d'écrire un précis de traduction se réclamant d'un statut scientifique; mais ils intitulent encore leur ouvrage Stylistique comparée du français et de l'anglais [1958]. $\gg^{14}$ Après eux, le professeur L. Bonnerot (Sorbonne) a publié en 1963 un ouvrage corollaire: Chemin de la traduction, domaine anglais (Paris, Didier). Voilà donc deux superbes ouvrages didactiques dont ne disposaient pas les traducteurs qui sont entrés dans le service avant 1958. C'est dire que, s'il y a aujourd'hui quelque trois cents traducteurs au service de l'État, peu d'entre eux ont eu l'occasion d'étudier ces ouvrages et que ceux-ci sont loin d'être entre les mains de tous les collègues. Rien ne me plairait autant, du reste, que d'être désabusé sur ce point.

Précisément, dans leur Stylistique comparée, MM. Vinay et Darbelnet déclarent que la méthode qu'ils proposent «ne s'applique pas uniquement aux travaux des professionnels, mais aux différents domaines de la traduction ... le domaine scolaire, le domaine professionnel et celui de la recherche linguistique ». Ils disent aussi: «On lit trop souvent que la traduction est un art ... En fait c'est une discipline exacte, possédant ses techniques et ses problèmes particuliers », et que c'est ainsi qu'ils entendent l'envisager.

Déjà au XVI $\mathrm{XV}^{\mathrm{e}}$ siècle, Étienne Dolet ${ }^{15}$ exprimait l'avis que «pour bien traduire, il faut avoir une parfaite connaissance de la langue de l'auteur et être pareillement excellent dans la langue dans laquelle on se met à traduire ». En 1953, Armand Pierhal faisait écho à ces paroles en disant: «... il faut que le traducteur possède non seulement la connaissance intime de la langue dans laquelle il écrit mais qu'il soit encore versé dans toutes les finesses de la langue qu'il traduit $\gg^{16}$. Ce jugement, cet axiome, ce truisme si l'on veut, MM. Vinay et Darbelnet le reprennent en précisant: «Quant au traducteur professionnel il doit

14. Georges Mounin, Les Problemes théoriques de la traduction, Paris, Gallimard, 1963, p. 8.

15. Cité par Edmond Cary, La Traduction dans le monde moderne, Genève, Georg \& C C', 1956, p. 40 .

16. Armand Pierhal, "L'art de la traduction ", La Parisienne, revue littéraire, avril 1953, p. 486 . 
connaître toutes les nuances de la langue étrangère et posséder toutes les ressources de sa langue maternelle. Autant dire que la grammaire et le vocabulaire ne doivent avoir aucun secret pour lui. ${ }^{17}$ Or, encore qu'au sens strict et dans la pratique ces terrifiants arrêts semblent prescrire un irréalisable idéal, ils ont sans doute pour objet d'indiquer le but vers lequel il importe de tendre. Le principe est du reste incontestable en ce qui concerne la traduction littéraire - celle d'un Scott-Moncrieff ${ }^{18}$ ou d'un Maeterlinck ${ }^{19}$ - mais on croira sans peine que dans le commerce, les affaires, la finance, l'administration, la presse d'information et certaines formes de la publicité, partout où l'on est censé faire «vite et bien », l'exigence stricte de pareilles qualités serait de nature à décimer les effectifs et à exclure à peu près tout aspirant.

Hans Vogt, spécialiste des études sur les contacts de langues, disait en 1954: «On peut aller jusqu'à se demander s'il existe un bilinguisme total, à cent pour cent. Cela signifierait qu'une personne puisse employer chacune de ses deux langues, dans n'importe quelle situation, avec la même facilité, la même correction, la même capacité que les locuteurs indigènes ... $\gg^{20}$.

Considérons un instant les propos de Graeme Ritchie et Claudine Simons, dans la Préface de leur Essays in Translation from French (Cambridge, 1952), recueil de 400 pages d'exercices de traduction littéraire:

«Translation» may mean many things, from loose inaccurate paraphrase upwards. The definition which we have adopted for our present (Academic) purpose is:

«Such a version as shall before all things make it plain that the translator:

1. has grasped the precise sense of each individual word or phrase as used

in the original,

2. has selected to render it, the nearest equivalent which English usage permits, and

3. has so arranged and welded together these equivalents that the whole becomes an exact counterpart of the French passage, equally careful in diction, equally elegant in style.»

Inutile de souligner qu'une norme aussi rigoureuse ne saurait guère être observée, et surtout maintenue sans aucune défaillance, par un traducteur fonctionnaire lorsqu'il est appelé, parfois durant plusieurs semaines d'affilée et au faîte de la canicule, à traduire de sept à huit mille mots par jour de prose délibératoire issue de l'autoclave parlementaire ${ }^{21}$.

La première consigne du traducteur fonctionnaire, c'est de viser à la fidélité, attendu qu'il travaille sur des textes officiels. En fait, et dans la pratique, serait-ce inadmissible de prétendre que les procédés de traduction puissent légitimement varier selon la nature des textes, leur destination, et le temps dont on dispose ? «Comment faut-il traduire telle lettre, tel document, tel rapport, telle formule,

17. Aussi: "... le traducteur doit connaître parfaitement la langue qu'il traduit et la langue vers laquelle il traduit $\gg$. (J.-P. Vinay, Journal des Traducteurs, vol. 2, no 4, oct.-déc. 1957, p. 143.)

18. La Parisienne, avril 1953, p. $484,492$.

19. Maurice Mreterlinck, La Tragédie de Macbeth, traduction nouvelle, Paris, Eugène Fasquelle, 1910.

20. Georges Mounin, op.cit., p. 6.

21. "... translators have freely contradicted one another about every aspect of their art, and ... they have written as if all translation were a conversion of a literary masterpiece in one language into a literary achievement in another $\gg$. (Theodore Savory, The Art of Translation, London, Jonathan Cape, 1957, p. 8.) 
tel projet de loi ?» Répondant à cette question, feu Edmond Cary la subdivise en trois autres questions: « QUE traduisez-vous ?», « où et QUAND traduisez-vous? 》 et, enfin, « POUR QUI traduisez-vous? ${ }^{22}$.

En définitive, ce qu'on demande au traducteur, ou à l'interprète, ce sont tout uniment l'omniscience et le parfait bilinguisme. Jean Herbert le dit bien: L'interprète parfait (lire aussi: le traducteur) serait une vivante et parfaite encyclopédie constamment tenue à jour de ce qui se fait et se dit dans tous les domaines. Cet idéal n'est évidemment pas réalisable mais l'interprète consciencieux doit inlassablement s'en rapprocher ... Il est indispensable que l'interprète ait des connaissances assez approfondies dans un certain nombre de sujets qui reviennent fréquemment (... histoire contemporaine, géographie politique et économique, droit civil, commercial et constitutionnel, économie politique, technique budgétaire, commerce international, procédure parlementaire, organisation internationale ...) et des notions plus superficielles mais précises dans des domaines aussi variés que la médecine, l'agriculture, les techniques des différentes industries, le fonctionnement des assurances, le droit maritime, la géographie physique, la sociologie, etc. ${ }^{23}$

Ailleurs, M. Herbert avait déjà dit:

L'interprète doit connaître à fond la langue à partir de laquelle il traduit ... Or pour connaître une langue, il ne suffit pas d'en posséder la syntaxe et le vocabulaire. Il faut aussi s'être imprégné de son esprit et de ses traditions, connaître ses sources et son évolution ancienne et récente, s'être familiarisé avec les auvres littéraires qui ont exercé sur elle une grande influence, apprécier son humour, connaître les noms qui ont jalonné son histoire, distinguer ses variantes stylistiques, être au courant de ses euphémismes, de ses délicatesses et des clichés dont le sens s'est atténué, parfois jusqu'à disparaître. Il faut aussi en connaître l'argot courant. L'interprète qui ne connaît pas bien la Bible, Shakespeare et même Bunyan et Lewis Carroll risque de se tromper lourdement sur le sens et la portée d'expressions employées en anglais. ${ }^{24}$

Hans Vogt, nous l'avons vu, doute de l'existence du bilinguisme à cent pour cent. Or il y a près de trente ans, Stephen Leacock exprimait une idée parallèle:

As a matter of fact, a full mastery of even two languages is a very rare thing. It can come only as the result of special environment, the opportunity to talk both, the will to do so, and therewith a certain aptitude. What is ordinarily thought as bilingualism falls away below this.

Et il ajoutait:

People who have devoted attention to the subject of foreign languages may be inclined to differ from me as to their valuation of translation. They may argue that translation represents as it were the last word, the supreme exercice in language. The extraordinary difficulty of finding idiom for idiom, of carrying over from one language to the other an absolute identity of meaning with an equal excellence of diction, such difficulty is only matched by the attraction of doing it. Now this is quite true. But such translation comes at the end, not at the beginning of study. ${ }^{25}$

$\mathrm{Au}$ Bureau des traductions, à Ottawa, la formation du groupe des traducteurs dits «stagiaires» se poursuit selon une formule qui réalise déjà un des

22. Edmond Cary, op. cit., ch. II, pp. 24-39. A ce même propos, on aurait tort de ne pas se reporter aussi aux pertinentes et savoureuses observations de Theodore Savory,
op. cit., p. 57,58 .

23. Jean Herbert, Manuel de linterprète, Genève, Georg \& C le, S.A., 1952, p. 22.

24. Jean Herbert, ibid., p. 14.

25. Stephen Leacock, Too Much College, New York, Dodd, Mead \& Co., 1939, p. 104, 105. 
vœux exprimés au Congrès de la FIT à Bad Godesberg en 1959 («systematic practical training under supervision, for at least one year ...») et comportant une proportion d'enseignement théorique.

Entre-temps, des spécialistes s'appliquent à mettre au point des machines à traduire. John Diebold écrivait tout récemment: «Work is being done on language translation by machine. Some document-translation is already on a regular production basis ... This work is still in its beginning stages and there are many problems to be overcome. But the history of this technology is that what seems impossible today becomes an accepted part of our lives tomorrow. $\gg^{26}$

Toutefois, Émile Delaveney disait, à Bad Godesberg:

Selon Bar-Hillel, la traduction pleinement automatique de haute qualité n'est pas réalisable dans un proche avenir, et ne l'est pas du tout à moins que des machines ne soient construites, et des programmes rédigés pour elles, qui dotent ces machines d'une intelligence quasi humaine ... Par contre le rigoureux critique qu'est Bar-Hillel reconnait que le moment est venu où des équipes commerciales de traduction dans lesquelles l'homme sera associé à la machine, peuvent devenir une réalité pratique ... Il faut cependant pour aboutir à des résultats économiques, posséder un lecteur direct de caractères d'imprimerie et faire l'effort collectif considérable nécessaire pour réaliser les dictionnaires électroniques indispensables. ${ }^{27}$

Deux ans plus tôt, Delavency avait écrit:

Qu'attend-on pour former des traducteurs? Il est vrai que c'est une tâche urgente pour nos écoles, tâche d'une longue haleine et souvent ingrate, imparfaitement conçue d'ailleurs sauf dans les rares institutions où le problème est posé avec réalisme, sans recherche prématurée d'effets littéraires. La traduction automatique n'est pas une raison pour relâcher les efforts des écoles; bien au contraire, elle aura aussi besoin de nombreux linguistes rompus aux meilleures disciplines du traducteur humain. ${ }^{28}$

En somme, la formation du traducteur, tout comme l'étude approfondie de même une seule langue, fût-ce la nôtre, est l'affaire de toute une vie ${ }^{29}$. Se former, c'est s'informer ... en s'abreuvant méthodiquement, continûment, aux sources d'un savoir reconnu. Ce qui serait déplorable, ce serait l'incurie, ou le refus de profiter de ce que - dans le seul domaine français — les instruments et les moyens d'étude n'ont jamais été si bons, si nombreux et si variés qu'à notre époque.

\section{DENYS Goulet}

Directeur du Centre de terminologie Bureau des traductions Secrétariat d'État, Ottawa

26. John Diebold, "The New World Coming», New York, Saturday Review, 23 juillet 1966, p. 17.

27. Emile Delaveney, La Qualité en matière de traduction (Actes du Congrès international de la traduction de Bad Godesberg, 1959), New York, The Macmillan Company, 1963, p. 313,314 .

28. Emile Delaveney, La Machine à traduire, Paris, P.U.F., «Que sais-je ?», 1959, p. 9.

29. Cf. Marcel Paré, «Le traducteur, étudiant pour la vie », Journal des Traducteurs, 1958, p. 67, et J. Darbelnet, loc. cit., 1963, p. 135. 